\title{
Extending WLAN Coverage via Utility Pipes
}

\author{
A. E. Xhafa and O. K. Tonguz ${ }^{\dagger}$ \\ Texas Instruments Inc., Dallas, TX 75243, USA \\ Email: axhafa@ti.com \\ ${ }^{\dagger}$ Carnegie Mellon University, Department of Electrical and Computer Engineering \\ Pittsburgh, PA 15213-3890, USA \\ Email: tonguz@ece.cmu.edu
}

\begin{abstract}
In this paper, we propose an inexpensive solution to the extension of wireless local area networks (WLAN) coverage. Our work shows that one can use antennas connected to the utility pipes (e.g., natural gas pipes, drinking water pipes, etc.) to extend the coverage of WLAN. Furthermore, this approach eliminates handovers from high to low data rate networks; e.g., handovers that occur from WLAN to a General Packet Radio Service (GPRS) network. Therefore, the proposed approach avoids the drastic drop in the data rate when handovers occur from high to low data rate wireless networks. We also develop an analytical framework to evaluate the performance of the proposed approach.
\end{abstract}

\section{INTRODUCTION}

To provide high-speed Internet access to mobile users, many different wireless local area networks (WLAN) have been installed in different areas such as coffee shops, airports, hospitals, etc. These networks, called hot-spots, are based on IEEE 802.11 technology and allow users to achieve physical data rates of $54 \mathrm{Mbps}$ or higher. However, the coverage areas of these hot-spots usually do not overlap; thus, their geographic coverage is poor. Improvement of the geographic coverage of these hot-spots can be achieved by extending the coverage of the existing hot-spots (or by installing new hot-spots) such that coverage areas of these hot-spots overlap. However, both of these solutions require acquisition of the right of way to lay down communication cables. This acquisition comes at an enormous first time cost.

To avoid the cost associated with acquisition of the right of way, in this paper, we propose a new approach. In this approach, antennas connected to the utility pipes are used to extend the coverage of WLAN networks (or hot-spots). Antennas connected to the utility pipes can communicate via cables, optical fiber, or coax cables, inserted in the utility pipes [1]-[4]. Extending the coverage of WLAN via utility pipes is an inexpensive approach.

Furthermore, next generation wireless networks will be composed of more than one basic link technology. Routing to mobile nodes should take advantage of this diverse connectivity to provide better geographic coverage and better network performance. However, to take advantage of this diverse connectivity, the system must deal with issues of vertical handover between different networks [5]-[7]. For example, the system must consider the following issue:

Handover between different network tiers (vertical handovers), for example, handover from a WLAN that supports
54 Mbps or higher data rate to a General Packet Radio Service (GPRS) network that supports $115 \mathrm{Kbps}$, can lead to a very different quality of service (QoS) available to the mobile terminal [5], [6]. Antennas connected to the utility pipes can be used to provide the communication link between the old base station (BS) to a mobile user (MU) that moves out of the coverage area of the high data rate networks; thus, reducing vertical handovers.

Next we briefly describe the concept behind using utility pipes to extend the coverage of WLAN networks and reduce vertical handovers.

\section{THE CONCEPT}

Handover between different network tiers can lead to a very different QoS available to the mobile users. Antennas connected to the utility pipes can be used to provide the communication link between the old BS to a MU that moves out of the coverage area of the high data rate networks. This idea is shown in Figure 1.

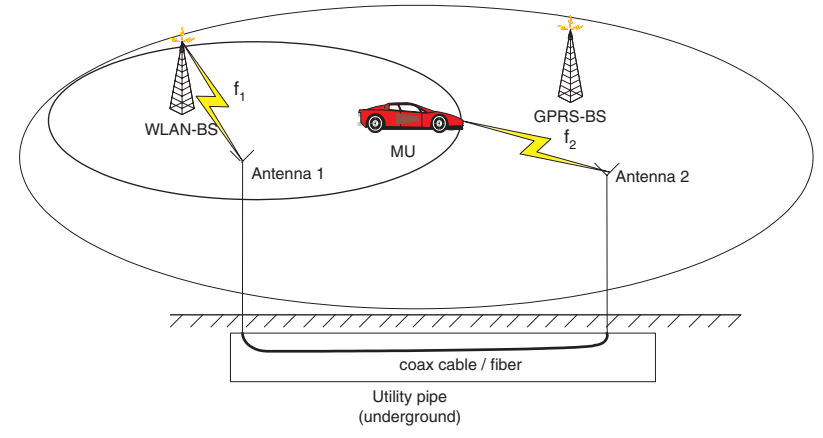

Fig. 1. Illustration of the use of utility pipes.

As the MU moves out of the coverage area of WLAN$\mathrm{BS}$, its data rate will reduce drastically. In order to avoid this, "Antenna 1" and "Antenna 2" are used to provide the communication link between the MU and WLAN-BS. The communication between the two antennas is done via the utility pipes. By providing the communication link between the WLAN-BS and the MU via the two antennas, the coverage area of the WLAN-BS is increased; thus, the number of handovers is reduced. In the case that the MU moves beyond the coverage area of "Antenna 2", another antenna can be coupled into the utility pipes to provide the communication 
link between the WLAN-BS and the MU. Coax cable or fiber could be used to provide communications between antennas in the utility pipe. Communication from MU/WLAN-BS to WLAN-BS/MU can be achieved in a few hops. It is possible that more than one antenna is needed to provide the coverage for the MU that moves out of the coverage area of WLAN-BS, so that a drastic data rate reduction does not happen. At any point in time though, the MU could switch to another network using the antennas connected to the utility pipes to relay this information to the WLAN-BS.

Next, we present an analysis to evaluate the performance of the proposed approach.

\section{AnAlysis}

In this section, we present a simple analysis to evaluate the performance of wireless networks that use utility pipe networks.

\section{A. Number of Antennas Needed}

In order to calculate the number of antennas needed, we refer to Figure 2. Assuming that from high data rate cell 1 to high data rate cell 2 , the mobile users are moving in a straight line, then, the number of antennas between the cells depends on the virtual cell (VC) radius $d$. In order to avoid the data rate degradation, the whole straight path between two cells has to be covered by antennas connected to the utility pipes. Using simple algebra, one finds that

$$
d=\frac{1}{2}\left(\sqrt{3} R+2 \sqrt{r^{2}-\frac{R^{2}}{4}}+\sqrt{3}\left(N_{A}-2\right) r\right)
$$

where $R$ is the coverage radius of the WLAN-BS, $r$ is the coverage radius of the antenna attached to the utility pipe, and $N_{A}$ is the number of antennas that is needed to cover the straight distance between the two cells. We have assumed that the arcs that make up the intersection area between the WLAN-BS and the first antenna cell span a cord of length $R$. It is clear that the coverage radius for the antennas, $r$, will depend on the power received at the mobile user terminal. The power received at the user terminal in addition to the noise power should be at least equal to the sensitivity needed at the mobile user terminal for a correct reception of the messages/packets from the antenna. Based on this constraint, one can calculate the coverage radius of the antenna. However, link budget analysis details will be omitted in this paper. Instead, we will focus our attention on the traffic analysis.

From Eqn.( 1) one gets

$$
N_{A}=\left\lceil\frac{2 d-\sqrt{3} R-2 \sqrt{r^{2}-\frac{R^{2}}{4}}}{\sqrt{3} r}+2\right\rceil
$$

Since we have calculated the number of antennas needed to cover the area between the two cells, we continue with handover performance of the proposed approach.

\section{B. Handover Rate}

We assume that the high data rate network, i.e., WLAN, consists of only one cell and there are no neighboring high data rate networks; otherwise, a handover does not lead to a drastic drop in the data rate for the mobile terminal. However, if one considers the $\mathrm{VC}$ with radius $d$, then, there is a

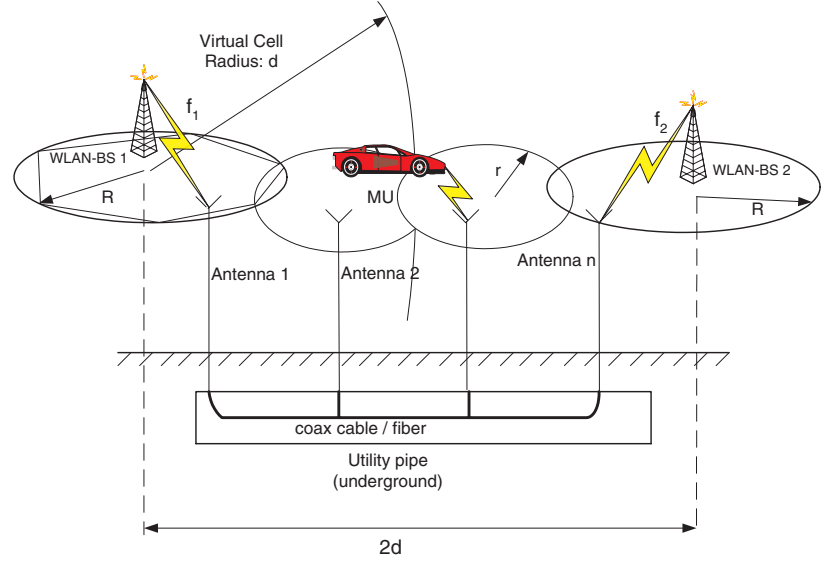

Fig. 2. Covering the area between two WLAN cells.

neighboring VC for the WLAN cell. Therefore, the handover rate is calculated with respect to the coverage area of the VC that is covered by the WLAN-BS and the antennas connected to the utility pipes, since beyond that the mobile terminal is not covered by the WLAN-BS of cell 1 .

Consider the scenario given in Figure 2. The hexagon in the figure (not drawn to scale) represents the original coverage of the WLAN. In order to avoid a drastic drop in the data rate when a handover is required for a MU that moves out of the WLAN, antennas connected to the utility pipes are deployed as shown in Figure 2. For simplicity, we have shown only a few antennas. The way that this arrangement works is as follows:

If a connection request is originated within the hexagon area of the WLAN coverage, then WLAN-BS will serve this request. If a connection request is originated outside of the hexagon area, then there are two possibilities:

1) The requests are generated in the area between the hexagon and the circle of radius $R$. In this case, these requests can not be handover calls leaving the WLAN, since the handover calls should have switched to the antennas connected to the utility pipes by the time that these calls reach the hexagon boundaries. At this juncture, there are three possibilities:

a) The requests are new connection requests from customers served by the WLAN. The network can decide to serve or block these requests. If these requests are served, then it is done so via antennas connected to the utility pipes (AUP).

b) The requests are new connection requests from customers served by neighboring lower data rate networks. Again, the network can decide to serve or block these requests. If these requests are served, then it is done so via AUP.

c) The requests are handover requests arriving from neighboring lower data rate networks. Although the network could choose to block these requests, since dropping of a call in progress is more problematic than blocking of a new call, the network should accept these requests. These requests are handled by WLAN-BS.

2) The requests are generated in the area that is out of the 
coverage of the WLAN and sent to the WLAN-BS via the AUP. Again, these requests can not be handover calls leaving the WLAN, since the handover calls should have switched to the antennas connected to the utility pipes by the time that these calls reach the hexagon boundaries. There are three possibilities for these calls, which are the same as mentioned in item 1.

When the traffic between the $\mathrm{VC}$ is in equilibrium, then one has the following:

$$
\lambda_{h_{\text {out }}}=P_{h_{\text {new }}}\left(1-P_{B}\right) \lambda_{n}+P_{h_{\text {hand }}}\left(1-P_{H}\right) \lambda_{h_{\text {in }}}
$$

where $P_{h_{n e w}}$ and $P_{h_{h a n d}}$ are the probabilities that a new and handover call will experience a handover, respectively; $P_{B}$ and $P_{H}$ are the new call blocking and handover failure probabilities, respectively; $\lambda_{h_{\text {out }}}$ and $\lambda_{h_{\text {in }}}$ are the handover rates out and into the $\mathrm{VC}$; and $\lambda_{n}$ is the new call originating rate within the WLAN-BS cell. In equilibrium one has $\lambda_{h}=$ $\lambda_{h_{\text {out }}}=\lambda_{h_{\text {in }}}$ and

$$
\lambda_{h}=\frac{P_{h_{\text {new }}}\left(1-P_{B}\right)}{1-P_{h_{\text {hand }}}\left(1-P_{H}\right)} \lambda_{n}
$$

where the total new request connection rate in the WLAN is:

$$
\lambda_{n}=\frac{3 \sqrt{3}}{2} R^{2} g
$$

where we consider all the connection requests within the hexagon area of coverage and $g$ is the call generation rate $/ \mathrm{min} / \mathrm{m}^{2}$.

New call blocking and handover failure probabilities are calculated in a similar way as the one described in [8], [9]. To calculate $P_{h_{n e w}}$ and $P_{h_{\text {hand }}}$ probabilities we consider different scenarios for new calls and for handover calls. We first consider the scenarios for the new calls.

$P_{h_{n e w}}:$ Scenario 1 . New calls that get service are generated only on the WLAN-BS hexagon cell.

First, we approximate the hexagon cells with the equivalent circular cells that have radii:

$$
\begin{gathered}
R_{e q}=\sqrt{\frac{3 \sqrt{3}}{2 \pi}} R \approx 0.91 R, \\
d_{e q}=\sqrt{\frac{3 \sqrt{3}}{2 \pi}} d \approx 0.91 d
\end{gathered}
$$

We assume that the residual time is a random variable, $T_{n}$, that follows an exponential distribution. The mean of the residual time is given as [8]:

$$
E\left[T_{n}\right]=\frac{E[Z]}{V_{\max }} \ln \left(V_{\max }\right)
$$

where $Z$ is a random variable denoting the distance of the MU to the VC boundary, while $E[Z]$ is its expected value [8], [9]. We have assumed that the MU is equally likely to move in any direction, his direction remains constant during his movement, and his speed is uniformly distributed between $1 \mathrm{~m} / \mathrm{hr}$ and $V_{\max }$. As it is difficult to obtain a closed-form expression for $E[Z]$ in this case, we compute it numerically for different values of $d_{e q}$ and $R_{e q}$.

The event that a new call will experience a handover is equivalent to the event that the residual time for the new call has a smaller time duration than the uninterrupted call duration, $T_{c}$. Assuming that $T_{c}$ is a random variable that follows an exponential distribution with mean $1 / \mu_{c}$, one gets:

$$
\begin{aligned}
P_{h_{\text {new }}} & =P\left(T_{n}<T_{c}\right) \\
& =\frac{V_{\max }}{\mu_{c} E[Z] \ln \left(V_{\max }\right)+V_{\max }}
\end{aligned}
$$

$P_{h_{\text {new }}}:$ Scenario 2. New calls generated out of the WLAN-BS region are served.

In this case, the average travelled distance by the new calls to reach the $\mathrm{VC}$ boundary is given as:

$$
E[Z]=\frac{8 d_{e q}}{3 \pi}
$$

We again assume that the residual time follows an exponential distribution, therefore, we are interested in the mean of the residual time. This is given as in Eqn.( 8), while the probability that a new call will experience a handover has the same expression as in Eqn.( 9).

To calculate the probability that a handover call will experience another handover call, which is equivalent to the event that the handover call residual time is shorter than the uninterrupted handover call duration time, we consider the following scenarios:

$P_{h_{\text {hand }}}:$ Scenario 1 . Only handover calls arriving from outside of the VC are considered.

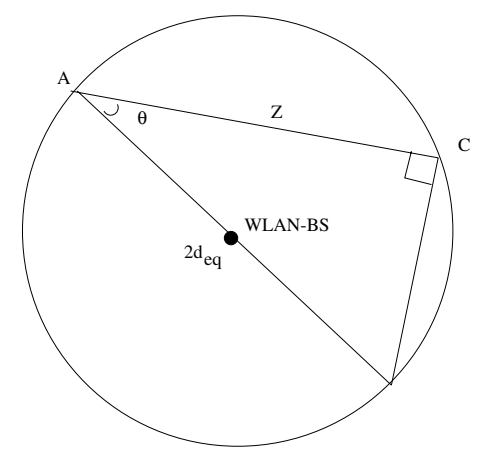

Fig. 3. Illustration of distance from point $\mathrm{A}$ in a cell, where the call enters the cell, to point $\mathrm{C}$ on $\mathrm{VC}$ boundary.

In this case, the analysis is similar to the one developed by Hong and Rappaport [9]. The expression for $P_{h_{\text {hand }}}$ is the same as that of $P_{h_{n e w}}$ given in Eqn.( 9); however, $E[Z]$ will have a different value since the scenario is different (see Figure 3). Looking at Figure 3, after some steps one finds that $E[Z]$ in this case is given as:

$$
E[Z]=\frac{4 d_{e q}}{\pi} .
$$

The expression for $P_{h_{h a n d}}$ is the same as that of $P_{h_{n e w}}$. Substituting for $E[Z]$, one gets:

$$
P_{h_{\text {hand }}}=\frac{V_{\max }}{4 \mu_{c} d_{e q} \ln \left(V_{\max }\right) / \pi+V_{\max }} .
$$

$P_{h_{\text {hand }}}:$ Scenario 2. All the handovers inside and outside of the WLAN-BS cell are considered.

We will assume that handover calls within the VC make a certain percentage of the total handover calls arriving in the cell. Note that the handover calls could also be generated 
inside the WLAN-BS; e.g., calls that want to improve their QoS. Hence, the handover calls that are generated within the $\mathrm{VC}$, can be treated as the new calls (see Scenario 2 for the new calls). Therefore, the overall average handover residual time can be calculated as the average of the residual time of the handover calls generated within the $\mathrm{VC}$ and those handover calls arriving from the neighboring $\mathrm{VC}$ cell. The average residual time for handover calls generated within the $\mathrm{VC}$ is the same as the average residual time found for the new calls generated within the VC cell (see Scenario 2 for calculation of $\left.P_{h_{\text {new }}}\right)$. The average residual time for the handover calls arriving from the neighboring $\mathrm{VC}$ cells, is the same as the average residual time found for handover calls in Scenario 1. Thus, assuming that the percentage of the handover calls generated within the $\mathrm{VC}$ cell is $p$, the average residual time for the handover calls in this case is given as:

$$
E\left[T_{h}\right]=p \frac{8 d_{e q} \ln \left(V_{\max }\right)}{3 \pi V_{\max }}+(1-p) \frac{4 d_{e q} \ln \left(V_{\max }\right)}{\pi V_{\max }}
$$

Assuming that the residual time $T_{h}$ follows an exponential distribution with mean given in Eqn.( 13), one finds

$$
P_{h_{\text {hand }}}=\frac{1}{1+\mu_{c} E\left[T_{h}\right]} .
$$

\section{Data Rate}

In order to calculate the impact of the AUP deployments on the drop in the data rate of mobile terminals, let us assume that the data rate/capacity provided in WLAN is $D$. Then, neglecting the interference, the data rate/capacity provided in the AUP regions is the one provided by the WLAN-BS. Hence, the VC is served by the WLAN-BS. As a result, the data rate provided to each MU decreases. The average number of calls being served in the WLAN region and the regions covered by the antennas is given as:

$$
N_{V C}=\sum_{n=0}^{N_{W L A N}} n p_{n}
$$

where $N_{W L A N}=\left\lceil D / D_{\text {min }_{u}}\right\rceil$ denotes the maximum allowable number of users in $\mathrm{VC}$ cell given a minimum data rate per user $D_{m i n_{u}}$, and $p_{n}$ denotes the probability that there are $n$ users being served in the system and it is given as [8]:

$$
p_{n}=\frac{\rho^{n}(1-\rho)}{1-\rho^{N_{W L A N}+1}}
$$

and $\rho$ is given as $\rho=\lambda / \mu$, where $\mu$ is the service time duration for a connection. Thus, the data rate provided to the users in VC cell is:

$$
D_{M U}=\max \left\{D_{\min _{u}}, \min \left(D / N_{V C}, D_{\max _{u}}\right)\right\}
$$

where $D_{\text {max }}$ denotes the maximum data rate that a mobile terminal can handle.

\section{RESUlts AND Discussion}

In this section, we present numerical results based on the analysis described in the previous section.

Figure 4 shows the number of antennas versus the distance between two WLAN cells and for different scenarios of $r$, where $r$ is the area covered by the antenna. It is clear that $r$ depends on the environment type. For example, for an open

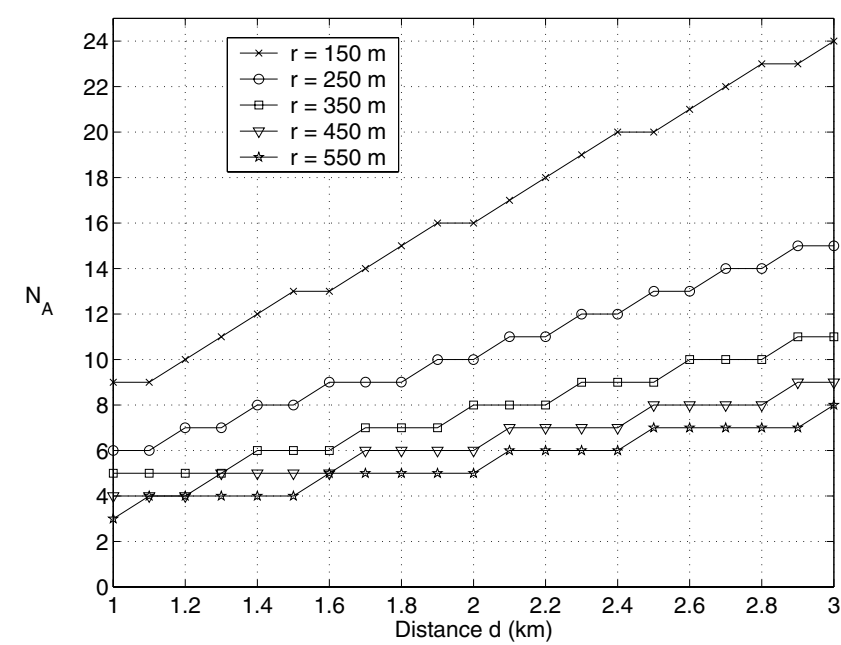

Fig. 4. Number of antennas needed versus distance between two WLAN cells.

environment, $1 \mathrm{Mbps}$ data rate can be provided for a distance of up to 550 meter, while the sensitivity of the MU receiver in this case is $-94 \mathrm{dBm}$. For a semi-open environment, the coverage radius drops to 115 meters. One can observe in Figure 4 that as the coverage radius for the antennas connected to the utility pipes increases, the number of antennas required decreases, which is expected. Since the antennas would operate in an open environment, then antenna radius coverage of 550 $\mathrm{m}$ would be a reasonable assumption. Hence, the number of antennas needed to cover, for example a distance of $3 \mathrm{~km}$ between two WLAN cells is 9 . Although number 9 might seem too many, the cost to install these antennas is much lower than that of base stations, which makes the proposed approach a cost effective solution.

To investigate the impact of the proposed scheme on the handover rate, we assume that the coverage area for the WLAN cell is 50 meters; the service rate for the calls is 0.1 calls/min; the maximum speed is $5 \mathrm{~km} /$ hour, while the radius of the VC cell is varied from 50 to 1000 meters. Figure 5 shows the reduction in the handover rate as the ratio of the handover rate in the $\mathrm{VC}$ and the handover rate in the original cell. In this case, we have assumed that the calls originating only within the WLAN-BS cell are served, while the new calls originating in the $\mathrm{VC}$ region are blocked; i.e., if a $\mathrm{MU}$ in the first antenna cell requests a new connection, this request is blocked. Furthermore, we also consider only the handover rates arriving from the neighboring $\mathrm{VC}$; hence, handover calls from low data rate neighboring cells are note considered. One can observe that as the distance between the WLANBS increases, the handover rate decreases, which is what one would expect.

Since multiple antennas could be used for WLAN extension, there exists handover when MU move from one antenna coverage to the next antenna coverage. This handover is intracell handover and it is handled by WLAN-BS since the antennas act simply as repeaters. If handover occurs between antennas that are connected to different WLAN-BSs, then, this handover is inter-cell handover and is handled by the WLANBSs.

Note that the traffic load in a cell is the sum of the 


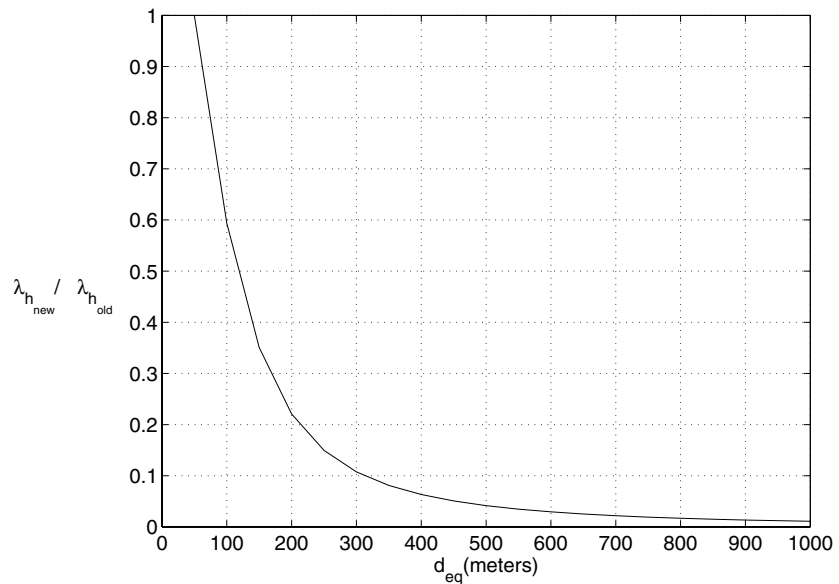

Fig. 5. The improvement on the handover rate when using antennas connected via the utility pipes.

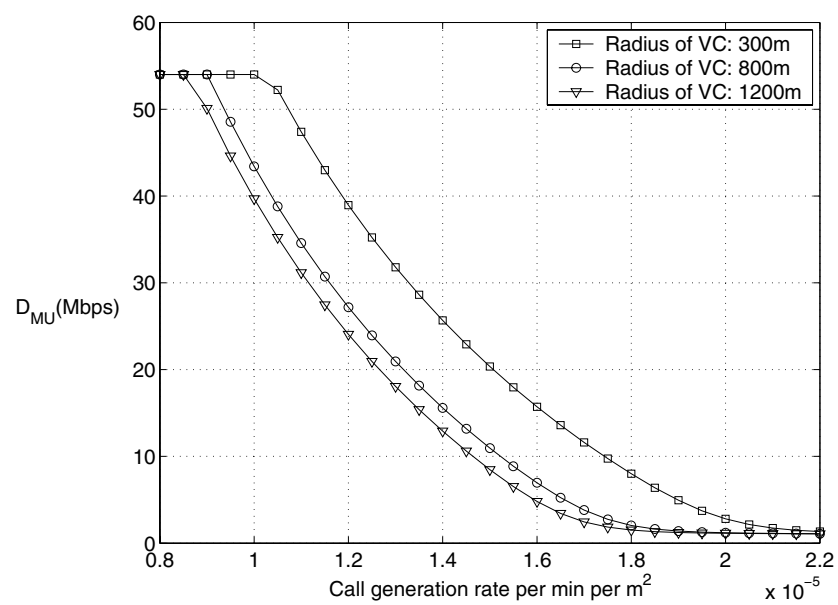

Fig. 6. Data rate provided to MU versus new call generation rate and for different VC radius coverage.

new generated calls and handover arrivals. If the new call generation remains the same, while the handover call arrivals decrease, this implies that the total traffic in the cell decreases; hence, one would expect that the data rate increases. However, as the $\mathrm{VC}$ radius increases, the channel holding time of calls also increases and its contribution to the offered load given by $\rho$ exceeds the decrease in the handover rate; i.e., for higher VC cell radius, $\rho$ is higher. It is also clear that as the new call generation rate increases, the data rate provided to each mobile user decreases. Figure 6 shows the impact on the data rate provided to the mobile users versus the new call arrival rate and for different scenarios; i.e., different VC cell radius coverage. As one can see, the data rate decreases as the new call generation increases, but for different $\mathrm{VC}$ cell radius coverage, the decrease is different. For example, for $\mathrm{VC}$ cell radius coverage of $300 \mathrm{~m}$ and $1.4 \times 10^{-5}$ per min per $\mathrm{m}^{2}$ call generation rate, the data rate is around $26 \mathrm{Mbps}$, while for $\mathrm{VC}$ cell radius coverage of $800 \mathrm{~m}$ and for the same call generation rate, the data rate is around $16 \mathrm{Mbps}$, which is 1.625 times lower. The reason is that as the $\mathrm{VC}$ radius increases, the channel holding time increases since calls already in progress are less likely to experience a handover. The increase in the channel holding time has a greater impact on the traffic load in the network than the decrease in handover traffic does. As such, the data rate provided to the mobile users decreases.

To compute the data in Figure 6 we assumed that the total data rate is $54 \mathrm{Mbps}$, the minimum data rate is $1 \mathrm{Mbps}$, the WLAN-BS cell radius is $50 \mathrm{~m}$, the service rate is 0.1 calls $/ \mathrm{min}$, and the maximum speed is $5 \mathrm{~km} /$ hour. It is clear that using utility pipelines to extend the coverage of WLAN networks, in addition to its low cost, it results in better overall network performance.

It is important to mention here that companies such as Alcatel, Corning, Sempra Fiber Links, as well as some of the owners of the utility pipelines are also exploring the use of deploying last mile fiber into the utility pipelines to provide high bandwidth to the end users [2].

\section{CONCLUSiOns}

We presented an innovative solution to extension of WLAN coverage. Our solution is based on the use of antennas connected to the utility pipes. This solution is inexpensive since a single utility is used for multiple purposes. Furthermore, this approach eliminates handovers from high to low data rate networks and thus, avoids the drastic drop in data rate for handovers calls "escaping" high data rate networks.

\section{REFERENCES}

[1] H. Nishikawa, T. Sasaya, T. Shibata, T. Kaneko, N. Mitumoto, S. Kawakita, and N. Kawahara, "In-pipe wireless micro locomotive system", in Proceedings of International Symposium on Mechatronics and Human Science (MHS'99), Nagoya, Japan, Nov. 24-26, 1999.

[2] J. K. Jeyapalan, "Municipal Optical Fiber Through Existing Sewers, Storm Drains, Waterlines, and Gas Pipes May Complete the Last Mile", 'ASCE Pipelines Conference, Baltimore, Maryland, USA: July 13-16, 2003.

[3] P. V. Nikitin, D. D. Stancil, A. G. Cepni, O. K. Tonguz, A. E. Xhafa, and D. Brodtkorb, "Propagation model for HVAC duct as a communication channel", IEEE Transactions on Antennas and Propagation, vol. 51, no. 5, pp. 945-951, May 2003.

[4] D. D. Stancil, O. K. Tonguz, P. V. Nikitin, A. E. Xhafa, and A. G. Cepni, "Assessment of Building HVAC Ducts as High-Bandwidth Communications Channels", CMU Technical Report, 2001.

[5] U. Varshney and R. Vetter, "Emerging mobile and wireless networks," Communications of the ACM, vol. 43, no. 6, pp. 73-81, June 2000.

[6] L. Taylor, R. Titmus, and C. Lebre, "The challenges of seamless handover in future mobile multimedia networks", IEEE Personal Communications Magazine, pp. 32-37, Apr. 1999.

[7] M. Stemm and R. H. Katz, "Vertical Handoffs in Wireless Overlay Networks" ACM Mobile Networking (MONET), Special Issue on Mobile Networking in the Internet, Winter 1998.

[8] A. E. Xhafa, "Analysis, design, and implementation of handover priority schemes in wireless networks", Ph.D. Thesis, Carnegie Mellon University, Thesis Advisor: Ozan K. Tonguz, Oct. 2003.

[9] D. Hong and S. S. Rappaport, "Traffic Model and Performance Analysis for Cellular Mobile Radio Telephone Systems with Prioritized and Nonprioritized Handoff Procedures", IEEE Trans. on Vehicular Technology, vol. VT-35, no. 3, pp. 77-92, August 1986. 